Lepr. Rev. (1970) 41, 57-61

\title{
The Role of Rehabilitation in Leprosy Control ${ }^{*}$
}

\author{
S. G. BROWNE, O.B.E. \\ Director, Leprosy Study Centre, London
}

Fashions change in medicine, just as they do in other spheres. Medical fashions are determined by scientific advances, public pressures and personal enthusiasms. Time was when it would have been unthinkable to use either of the terms "rehabilitation" and "control" in reference to leprosy, for the leprosy victim could look forward to nothing brighter than the merciful Lethe of death, and leprosy itself was not considered to be amenable to measures successfully applied to transmissible diseases in general. The leprosy sufferer could not be "rehabilitated", and the disease did not lend itself to "control".

Today we can, with some measure of justification, refer in the same breath to the rehabilitation of the individual and the control of the disease, but it would be idle to suppose that these two ideas in practice belong to the same conceptual world, or share the same vocabulary and techniques. In several respects, to change the figure, the reconstructive surgeons have all too few dealings with the epidemiologists, and prosthetists have even fewer with the therapists. Their backgrounds and objectives are different, and their approach to leprosy in the individual and in the community shows considerable divergencies. They speak different languages, and employ different techniques to reach different goals.

It will be our contention in this paper that the moment has come for the objective evaluation of the rôle of rehabilitation in leprosy control. If this evaluation is not begun soon, two dangers will become more apparent than they are today: first, medical planners and

*A paper given at the Eleventh World Congress of the International Society for Rehabilitation of the Disabled (I.R.S.D.), Dublin, September, 1969. administrators may decide, mainly on economic grounds, that poor developing countries simply cannot afford to include in leprosy control measures expensive schemes for the rehabilitation of leprosy patients; and second, isolated and non-integrated schemes for such rehabilitation-depending for the most part on private initiative, and the drive of dedicated individuals -will continue to provide the services for an overprivileged minority, while the leprosy endemic remains unchecked and preventable disabling deformity continues to occur.

A summary glance at recent history will clarify the reasons for the present situation. Until 25 years ago, the serious forms of leprosy were generally considered to be untreatable and inexorably progressive, deformity was the rule, and the idea of rehabilitation could not be entertained, either on social or on medical grounds. In the typical "Home" in India, devoted Christian missionaries laboured with self-sacrificing zeal and love, ministering to the hopelessly deformed and blind and outcast sufferers from leprosy or its sequelae and aftermath. While here and there some attempt at occupational therapy was made, no real vocational rehabilitation was contemplated, or, indeed, considered possible. There they were, and there they stayed. Cure of active serious leprosy was not on the agenda, and the physical deformities of leprosy were thought to be in some way unique and inevitable and not amenable to the standard surgical techniques employed, say, in poliomyelitis, or in trauma and infection of bones and soft tissues.

That situation is now outmoded, in two respects: first, medicinal treatment has become available that has transformed the outlook of the individual patient and has made possible 
the control and eventual eradication of leprosy; second, Paul Brand and others have shown conclusively that the principles of reconstructive surgery can be successfully applied to the deformities that result mainly from peripheral nerve damage in leprosy. In addition, scientifically established knowledge in many aspects of leprosy-in particular, its low infectivity and its curability-is slowly penetrating the offices of the health planners and the lecture halls of the medical schools. But the uncertainty and hesitation regarding the confusing and competing claims of leprosy control and leprosy rehabilitation continue unabated. The situation has not been clarified, either, by the advocacy of the representatives of the old status quo of "Homes" and asylums and the impatience of the champions of mass treatment. Furthermore, the trumpets of international agencies have given an uncertain note that has not prepared the willing warriors for battle.

\section{THE PRESENT POSITION}

Of the 15 million leprosy sufferers in the world only some 3 million are having treatment. A total of about 4 million have some physical deformity or sensory deficit attributable to leprosy. An unknown number, amounting probably to 3 millions or more, are in need of rehabilitation in some form or other. In general, where leprosy work has been established longest and has the most impressive buildings, the old outlook tends to persist. Within the institution, the care of the patients may be exemplary, and some occupational therapy may be given, but there has been little or no impact on the prevalence of leprosy in the surrounding villages. It is only of recent years that many such "Homes" are becoming centres for the purposes of leprosy control of an area. On the other hand, the mass treatment schemes spread thin over vast areas, are making deep inroads into the leprosy endemic in the areas concerned, but they offer no formal rehabilitation to the crippled or handicapped ex-patient: since he is no longer contagious, the mass treatment campaign tends to ignore him and his personal problems.

\section{THE PLACE OF REHABILITATION} IN A CONTROL PROGRAMME

We will accept the WHO comprehensive definition of rehabilitation as applied to leprosy: "The physical and mental restoration, as far as possible, of all treated patients to normal activity, so that they may be able to resume their place in the home, society and industry".

As I see it, two complementary conceptions have to be "sold" to two different kinds of government planning services and of medical units (whether the latter be separate leprosy institutions or polyvalent medical services). The first does not concern us primarily here, but must be mentioned for completeness' sake; it is, to persuade institutions where the emphasis has been disproportionately accorded to in-patient care and reconstructive surgery that these measures can only be justified as they are part of a leprosy control programme, integrated as far as possible into the overall policy for the control and treatment of transmissible diseases. That this is no theoretical consideration may be gathered from the observation that, in more than one country, schemes for leprosy control are languishing because workers (especially, let it be admitted, those working under voluntary agencies) have been concentrating latterly too much on dramatic and fashionable and professionally gratifying reconstructive surgery to the detriment of leprosy control. Leprosy sufferers, with progressive (and contagious) disease, are themselves developing deformities that are in the main preventible by adequate medical treatment, and they are at the same time transmitting leprosy to susceptible contacts. Patients with stigmatizing deformities will walk miles to seek surgery at distant shrines far from the peering eyes and gossiping voices of their own families and neighbours. Physiotherapy and surgical operations and education may together provide the basis for rehabilitation; but, on the other hand, impressive lists of operations performed at such a centre may bear little or no relation to the actual rehabilitation of the series composed of individual patients. At the "receiving end", in point of fact, and in the absence of a 
leprosy control service, the result may well be a confirmation that "leprosy can be cured by operation" and that this is all there is to it. True rehabilitation, including reconstructive surgery, must be part of a leprosy control programme, or it may fail in the long run as well as in the short.

A further point concerns the creation and development of medically excellent facilities that are unfortunately out of touch with, and divorced from, the whole life of the people and official medical policy. While it may be difficult to resist professional pressures for ever-rising (and progressively expensive) standards of accommodation and equipment, the resources of the country should not be overstrained in the interests-legitimate though they be-of a small section of the population. If $\$ 80$ per head per year were available, and not $\$ 1$ - for all health services-bigger and better rehabilitation services for all, including leprosy patients, could be provided.

The other complementary conception that has to be "sold" to the government planners and the voluntary agencies engaged in the antileprosy campaign is the one that concerns us principally here; viz., that rehabilitation really forms an essential component of any leprosy policy and project, that a well-thought-out plan is generally feasible, that it need not be prohibitively expensive, and that it possesses considerable advertising value.

\section{ESSENTIAL}

"Rehabilitation" in the conventional sense should ideally not be necessary if under a leprosy control scheme every person suffering from leprosy is detected early and treated adequately through a domiciliary service, which may (or may not) be integrated into general public health measures. Thus, if society does not discriminate against the leprosy patient, the patient will never be in need of rehabilitationfor physical, psychological or social reasons. $\mathrm{He}$ will not become deformed, since nerve damage is not the inevitable accompaniment of or sequela of leprosy; he will not become psychologically disrupted or isolated, since the environment will not necessarily induce such an attitude; and he will not be socially dislocated, because he will continue to be accepted as a member of the family, the village, the guild and the caste.

However, where this ideal of early diagnosis and adequate treatment is not yet attained, the idea of rehabilitation must be introduced from the outset of treatment, and everything done to instil the principle of prevention-not only prevention of deformity, but prevention of the mental attitude that will make rehabilitation in some form or other necessary. To this end, the doctor in charge and all those who work with him must be enthusiasts, and informed and competent enthusiasts at that. It goes without saying that treatment must follow diagnosis immediately, and that education goes hand in hand with treatment. The grouped patients are instructed in the care of insensitive extremities, how to avoid burns, cuts and abrasions, and damage by stones and thorns and nails in footwear.

The trained medical auxiliary accompanies the doctor and other team-members on survey and treatment journeys, and not only examines the patients for damage to vulnerable hands and feet, but also takes the opportunity to instruct the patients. Sometimes, plaster casts are applied on the spot, or protective footwear distributed, or sponge-rubber fixed to implements so as to reduce damage by friction and pressure.

Some patients, discovered on the first casefinding survey, already need more than "firstaid" or prevention. They are directed to or brought to the central hospital, where their special needs can be dealt with. The whole population can see that, whatever the stage leprosy has reached, the individual can be helped back towards normality. The stigma of the disease is slowly dispelled; false notions about the inevitability of deformity, the contagiousness of the discharge from neuropathic ulcers, the essential chronicity of such ulcers, and the uniqueness of leprosy in all its aspects, are gradually undermined. 
Unless some kind of rehabilitation service is grafted on to leprosy control measures, sooner or later the backlog of mutilated sufferers will become vocal and demand the impossible, restoration of function of lost digits and grossly deformed feet.

\section{FEASIBLE}

The actual sophistication possible in such a service depends mainly on finance: how much money can be made available, due consideration having been given to an objective evaluation of the benefits likely to be derived from the proposed service. Leprosy is only one of the crippling diseases-although it may be the most serious in point of numbers. Those handicapped by leprosy cannot really take precedence over others (despite their appeal on humanitarian grounds), and the crippledespecially if ageing - cannot demand a disproportionate share of available resources.

Government planners are understandably unco-operative when they are presented with blueprints for costly installations for rehabilitation requiring a large slice of the medical budget and staff for upkeep. Reconstructive surgeons, physiotherapists, occupational therapists, prosthetists, medico-social workers are all in short supply in developing countries, and they are expensive. But an installation suited to the Western world, and appealing to the Western-trained medical worker, may be quite out-of-place and ill-adapted to the needs and possibilities of a country with a huge leprosy problem.

\section{INEXPENSIVE}

The answer is severely practical: cut your coat according to your cloth, having made sure that the cloth is the best you can afford, the most hardwearing and best suited to the climate and the people. In other words, the rehabilitation service may begin simply with a polycompetent medical auxiliary attached to the leprosy survey team and to the domiciliary treatment scheme. His job is the prevention of deformity and education (in its broadest sense) in the setting of the family and the farm, or the community and the town.

With more money, the staff can be increased, and simple treatment given (such as plaster casts), simple operations performed (e.g., sequestrectomy), and simple protective footwear supplied (made from locally available materials, acceptable, cheap, long-lasting and repairable). Some patients with longstanding deformity require more than treatment given in the course of infrequent visits by the mobile team. Facilities for in-patient treatment should be provided in conjunction with the all-purpose rural dispensaries, the medical assistants in charge having had some instruction in leprosy and its management. This practical co-operation between the leprosy control service and rehabilitation and the general public health service demands little in the way of extra finance or specialized staff.

The next requirement is the creation of a rehabilitation unit where reconstructive surgery can be performed. Here again, it is preferable from many standpoints to include leprosy patients (or ex-patients) within the overall scheme. There is in general no need for separate institutions, though it is readily admitted that some specialized knowledge and constant practice are necessary if highly sophisticated surgical treatment is contemplated. However, much may be done by general surgeons, or general orthopaedic surgeons, if they have the time, the inclination and the facilities for pre- and post-operative physiotherapy, and the cooperation of makers of splints and footwear, and occupational therapists with local knowledge and the right outlook.

It is in this respect that the vision and initiative and flexibility of voluntary agency staff come into the picture. Provided that their work can be integrated practically into the leprosy control service, perhaps over a wider area than that immediately under the purview of the local control officers, then the additional burden on government funds should not be excessive. Central workshops for mass production of protective footwear, central schools 
for giving medical auxiliaries the extra specialized knowledge they need; and a modest central institute for correlation of records and research, may in the long run amply repay the investment. If the dangers of over-sophistication are avoided, and the staff continue to keep in touch with the needs of the average patient, then costs are kept down and standards kept up.

The value and practicability of vocational rehabilitation in developing countries faced with many urgent medical and social problems are matters for discussion. The nearer to the patient's life and livelihood such schemes are, the greater their chance of making a real contribution to rehabilitation. Thus, model farms are admirable: despite his handicaps, the leprosy ex-patient becomes a better and more successful farmer, after a short course here, than his healthy fellow-villagers. Soil preparation, composting, fertilizers, grafting, seed selection, contour ploughing, market gardening can all be taught to the great advantage and profit of the patients whatever the disease that caused their disability. The breeding of chickens, rabbits, goats, pigs, etc., may provide a good living.

In general, separate villages for ex-patients are not to be recommended, just as segregation villages often serve to perpetuate false notions about leprosy. An ex-patient who has some economic advantage to bring to the village is usually welcome, despite his deformity and despite the stigma attaching to the disease he has had.

\section{THE PROPAGANDA VALUE}

At every stage of the leprosy control programme, rehabilitation offers considerable propaganda or advertising advantages. It brings back the leprosy patient not only into the family, but also into the economic community. It helps to convince reluctant patients that something definite can be done for even the serious deformities caused by leprosy. And rehabilitation serves as a stimulus to unveiling of concealed leprosy: patients at earlier stages are brought out of hiding when their relatives see others who are managing to cope successfully with life despite the incubus of past leprosy. The sight of established deformity, now partially remedied, may be used as a salutary warning to patients who neglect to take treatment regularly.

Any measure that helps the leprosy control campaign to function more effectively should be welcomed. Rehabilitation is one of these measures, and not the least in importance or significance. 